\title{
Comparison of Sperm Parameters in Patients with Infertility Induced by Genital Infection versus Varicocele
}

\author{
Bogdan Pajovic ${ }^{1}$, Antonio Dimitrovski ${ }^{2}$, Nemanja Radojevic ${ }^{3}$, Marko Vukovic ${ }^{1}$ \\ ${ }^{1}$ Department of Surgery, Montenegro University Faculty of Medicine, Podgorica, Montenegro \\ ${ }^{2}$ Clinic of Urology, Clinical Centre of Kragujevac, Kragujevac, Serbia \\ ${ }^{3}$ Department of Forensic Medicine, Montenegro University Faculty of Medicine, Podgorica, Montenegro
}

Background: Male infertility is a common and complex problem and, despite much research in this field, the major cause of infertility unfortunately remains unknown. Genital infection and varicocele are important causes of infertility.

Aims: To compare the influence of genital infection and varicocele individually on male infertility based on semen analysis.

Study Design: Cross-sectional study.

Methods: The study included 120 infertile patients divided into two groups according to the presence of genital infection or varicocele. The first group included 60 examinees with proven genital infection, but without varicocele formation. The second included 60 patients with varicocele, regardless of the varicocele grade, but without genital infection. The fertile parameters were compared and an assessment was performed on the im- pact on quality of spermatogenesis due to infection and varicocele.

Results: There is a statistically significant difference regarding abnormal forms of spermatozoids $(45.94 \pm 9.79$ vs. $25.27 \pm 6.54)$ and progressive motility $(8.15 \pm 1.24$ vs. 24.95 \pm 7.2), between two groups of patients. However, acidity of ejaculates, minimum sperm concentration, total spermatozoid motility and ejaculate volume showed no statistically significant difference.

Conclusion: The study showed a stronger negative influence of genital infection on fertile parameters over varicocele. The significance of our study is the lack of contemporary researches comparing varicocele and genital infection influence on male infertility individually.

Keywords: Genital infections, infertility, semen analysis, varicocele
Infertility is related to the disability of a sexually active, couple not using contraception to become pregnant within one year. Male infertility is a complex, contemporary problem, which affects approximately every $20^{\text {th }}$ person. The etiology of infertility usually remains unknown, despite detailed and comprehensive research in this field (1-3).

Genital infection and varicocele are the main causes of male infertility worldwide. Genital infection can impair the quality of spermatozoa and decrease the function of male accessory glands. Nevertheless, it is a correctable cause of male infertility (4). Contemporary studies claim that varicocele can be associated with significant and progressive testicular damage if started during adolescence, which can permanently decrease fertility. Nevertheless, only a small percentage of men with varicocele are infertile, although almost $30 \%$ of men with abnormal semen parameters have clinical varicocele $(5,6)$. Initially, the diagnosis of infertility in males is established through the analysis of semen (7).

Since there is no contemporary research with regards to the comparison between genital infection and varicocele influence on male infertility, the aim of our research is to compare genital infection and varicocele influence on male infertility, separately, based on semen analysis. 


\section{MATERIALS AND METHODS}

The study included 120 infertile patients divided into two groups according to the presence of genital infection or varicocele, for the period between 2008 and 2013. The first group consisted of 60 examinees with a proven genital infection but without a varicocele formation. The second group included 60 patients with different grades of varicocele, but without genital infection. All patients had a minimum of a three-year history of infertility. We compared the fertile parameters and conducted the assessment of infection and varicocele impact on the quality of spermatogenesis. Microbiological tests were used for determining the presence of genital infection. We did not observe effects of surgical or medication treatment amongst the two groups of patients, since the emphasis was on pre-treatment values of semen analysis. Physical examination for varicocele presence was performed according to the criteria of the European Association of Urology (EAU) Guidelines on Male Infertility (5) and confirmed by color Doppler ultrasound.

We included subclinical varicocele patients in our study because of their impaired semen analysis (sperm count, total and progressive motility and abnormal forms), accompanied with a three-year infertility period. These occasions make them equal to those patients with clinically present varicocele, regarding the criteria of our study. Besides that, there are studies that indicate the influence of subclinical varicocele on male infertility (8-10).

We used a color Doppler flow imaging system and 7.5 MHz linear-array transducer (Hewlett-Packard-Sonos 1000; Providian Medical Equipment LLC, Willowick, OH, USA). The 7.5 MHz transducer was applied to the patient transscrotally. Color Doppler is the method of choice for detecting spermatic vein reflux and for classifying the grade of varicocele, regarding World Health Organization (WHO) 2000 guidelines on male infertility which state that a varicocele diagnosis should be confirmed by color Doppler (5). The contemporary review (11) shows that color Doppler is currently the most widely employed diagnostic method for detecting and classifying varicocele caused by venous reflux, as it is reliable and easily performed. Both testicular units were examined in supine and upright positions and during normal inspirium and Valsalva maneuver. Then the color imaging was done by placing the Doppler probe in the direction of the flow in the spermatic vein under the guidance of visible flow (12). A Doppler ultrasound criterion for varicocele is present in the vein in diameter from $3 \mathrm{~mm}$ or greater (13).

\section{Sperm analysis}

After 5 days of sexual abstinence, patients provided a sperm sample through self-administered masturbation, col- lected into sterile sample cups without the use of a lubricant. Samples were taken in our facility. The sperm samples were analyzed using a Sperm Quality Analyzer (SQA IIC-P; Medical Electronic Systems, Perchtoldsdorf, Austria), after 20 minutes of liquefaction. Sperm concentration, progressive motility, total motility, viability and morphology were determined. The reference values used, with the lower reference limit ( $95 \%$ confidence intervals) of the above-mentioned semen parameters, were taken from the criteria provided by the EAU guidelines (5).

\section{Volume and acidity (pH)}

The volume and $\mathrm{pH}$ of the fresh samples were determined according to our previous research (7), with the usage of digital pH-meter (the Extech EC510; Extech Instruments Corporation, Long Branch, NJ, USA) for acidity determination.

\section{Microbiological analysis}

This analysis was performed according to our previous study (7), where we used the polymerase chain reaction (PCR) technique on a portable PCR system (Palm PCR F1-12, California, United States), for antigen detection. For the purpose of this research, there was no separation of examinees in subgroups according to the species involved.

\section{Inclusion criteria}

The first group included patients with a history of infertility which lasted for at least three years with an abnormal semen analysis and painful varicocele, with no concomitant urological diseases. The second group included patients with infertility that lasted for at least three years, had a positive urethral smear along with a positive microbiological semen sample and no other concomitant urological disease.

\section{Exclusion criteria}

Since newer studies indicate that smoking influences fertility parameters, we excluded all persistent smokers and those who were temporary smokers. We also excluded patients who experienced alcohol abuse. Patients with chromosomal abnormalities, sperm autoantibodies, testicular tumors or those with systemic diseases were not included in our study. Diabetic patients and those with cystic fibrosis or who had undergone a vasectomy were also excluded from this research.

\section{Ethics}

Each subject signed the acceptance of the study protocol, in which the Ethical Principles for Medical Research Involving Human Subjects (The Helsinki Declaration) were clearly stated. 


\section{Statistical analysis}

Statistical analysis has been performed by the Statistical Package for the Social Sciences (SPSS) version 11 for Windows software package (SPSS Inc., Chicago, USA). Methods of statistical description included the $\mathrm{Z}$ test for proportion and the Student $T$ test, in order to determine statistical significance. The difference of the values obtained was considered to be significant when $\mathrm{p}<0.05$, and highly significant when $\mathrm{p}<0.01$.

\section{RESULTS}

The first group included 60 examinees with proven genital infection, with an average age of $33 \pm 4.5$ (ranging from 25 to 50). Table 1 provides the frequencies of common causes of genital infection. During the comparison of the semen parameters with the group of patients with varicocele, there was no sub-grouping regarding the type of infective agent.

The second group entailed 60 patients with varicocele formation, with an average age of $30 \pm 5.5$ (ranging from 22 to 53). Table 2 shows different distributions of varicocele grade amongst the second group of patients. By comparing semen parameters with the first group, there was no sub-grouping regarding the varicocele grade. We evaluated homogeneous groups and no statistical difference was found for age.

According to the data presented in Figure 1, there was no significant difference in the acidity of ejaculates between the two groups of patients ( $p>0.05)$, nor regarding ejaculate volume ( $p>0.05)$.

Data from Figure 2 and Figure 3 clearly reveal a highly statistically significant difference in progressive motility and abnormal forms of spermatozoids $(\mathrm{p}<0.01)$ amongst those two groups of patients, unlike sperm concentration and total spermatozoid motility which are not significantly different $(\mathrm{p}>0.05)$.

\section{DISCUSSION}

Our study shows a stronger negative influence of genital infection on fertile parameters over varicocele. There was a highly statistically significant difference regarding abnormal forms of spermatozoids and progressive motility. Nevertheless, there were no significant differences in ejaculate volume, sperm concentration, ejaculate acidity and total spermatozoid motility between those two groups. Although it was noticed that the values for total spermatozoid motility were lower in infertile examinees with varicocele compared to those for in-
TABLE 1. Distribution of bacterial species present in the first group of patients

\begin{tabular}{lc}
\hline Microorganism & Number of patients \\
\hline Chlamydia trachomatis & 20 \\
Neisseria gonorrhoeae & 17 \\
Mycoplasma genitalium & 8 \\
Trichomonas vaginalis & 6 \\
Ureaplasma urealiticum & 4 \\
Micoplasma hominis & 3 \\
Herpes simplex virus & 2 \\
\hline
\end{tabular}

TABLE 2. Frequency of different varicocele grades among the second group of patients

\begin{tabular}{lc}
\hline Varicocele grade & Number of patients \\
\hline Subclinical varicocele & 23 \\
Grade 1 & 18 \\
Grade 2 & 10 \\
Grade 3 & 9 \\
\hline
\end{tabular}

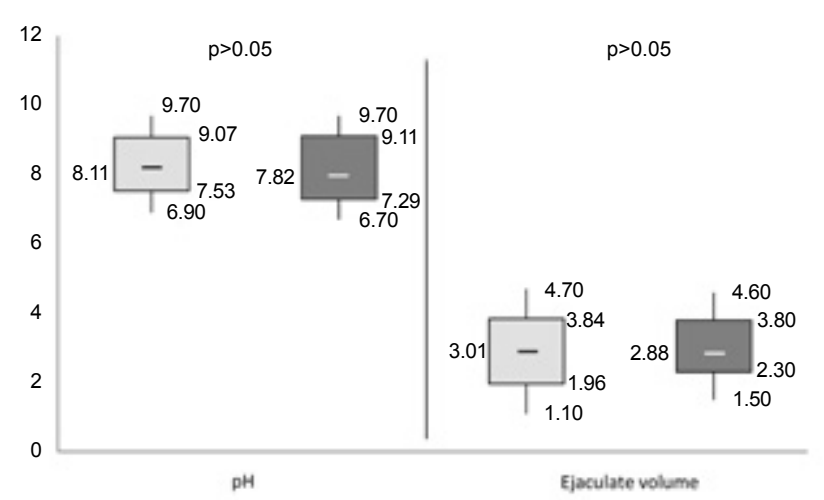

FIG. 1. Comparison of $\mathrm{pH}$ and volume of ejaculate between two groups of infertile patients

Light grey boxes represent the mean $\pm S D$ of the group of patients with genital infection while horizontal lines represent average value and lines show the minimum and maximum. Dark grey boxes represent the mean $\pm S D$ of the group of patients with varicocele, and lines indicate the minimum and maximum.

fertile examinees with genital infection, as well as ejaculate volume and the number of spermatozoids in ejaculate, the study clearly shows a better outcome of varicocele on semen and fertile parameters than genital infection.

Many contemporary studies $(7,14,15)$ concerning the correlation between pyospermia or leucocytospermia and decreased semen parameters. Those authors claim that asymptomatic pyospermia has great influence on male infertility, with significant occurrence, and it can reflect infectious or inflammatory disorders. Nevertheless, the role of specific genital tract infections is crucial. The research of SanockaMaciejewska et al. (16) showed significant deterioration in 


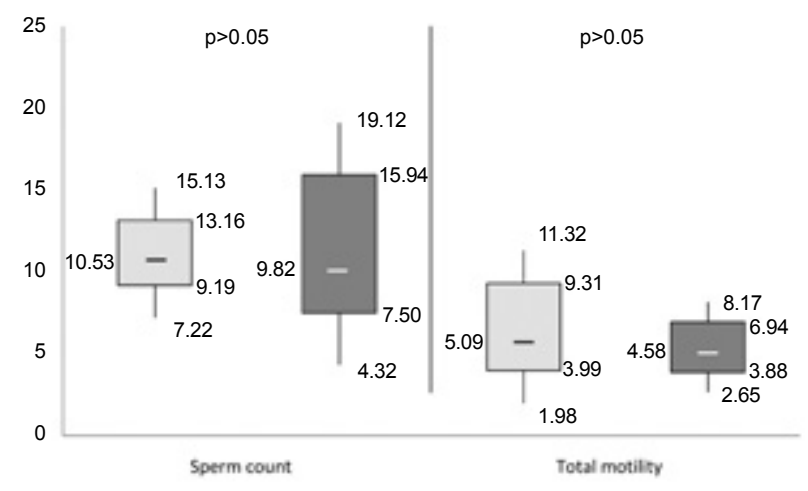

FIG. 2. Comparison of sperm count and total motility between two groups of infertile patients

Light grey boxes represent the mean $\pm S D$ of the group of patients with genital infection while horizontal lines represent average value and lines denote the minimum and maximum. Dark grey boxes represent the mean $\pm S D$ of the group of patients with varicocele, and lines indicate the minimum and maximum.



FIG. 3. Comparison of progressive motility and abnormal forms between two groups of infertile patients

Light grey boxes represent the mean $\pm S D$ of the group of patients with genital infection while horizontal lines represent average value and lines indicate the minimum and maximum. Dark grey boxes represent the mean $\pm S D$ of the group of patients with varicocele, and lines denote the minimum and maximum.

almost all parameters of semen in patients with genital tract infection in comparison to healthy controls, but without a statistically significant difference compared to the infertile group without infection. Unlike in our study, they did not precisely state the cause of infertility in the second group of patients, nor did they investigate varicocele influence on male infertility. A study made by Ma et al. (17) indicated a significantly decreased sperm concentration, sperm progressive motility, and sperm viability, but there was no remarkable difference in semen volume and non-progressive motility between infertile patients with varicocele and normal donors in the control group. Their study did not include patients with genital infections, however, so the study could not establish a comparison between the two major factors of male infertility, unlike our study. Abdulmedzhidova et al. (18) examined infertile patients with and without Herpes simplex virus (HSV) infection in their study. In the comparison group, there were patients with varicocele, including patients with HSV. Their research showed that there is no significant difference between the majority of sperm parameters in the HSV-positive and HSV-negative groups. In contrast to this study, our research showed that there is a significant difference between a few mean values of sperm parameters and no difference between ejaculate volume, total motility and sperm concentration amongst the two groups of infertile patients, which implies that there is a better prognosis in patients with varicocele but without genital infection. As we can see, their study did not discuss varicocele influence on fertility parameters or the relation between infection and varicocele itself, which makes this study insufficient in the matter of our research. A study performed by Li et al. (19) compared the influence of genital infection (Ureaplasma urealyticum) and varicocele with genital infection together on fertility parameters. In this research, they showed that asthenozoospermia was significantly more prevalent in the patients with varicocele than in the other patients and a significant quantitative difference in the incidence of genital infection between the varicocele and control group was observed. This study, however, did not include any other fertility parameters, except asthenozoospermia, and did not show the influence of varicocele and genital infection individually on male infertility and the comparison of its influences, as our study did. There is no cotemporary research clearly comparing varicocele and genital infection influence on male infertility individually; thus, our study can be considered important material for future investigation and the treatment of this problem. The limitations of our study include a lack of further comparison (after obtained treatment) of those two infertility issues, as well as their followup and pregnancy rate incidence after the performed treatment. Although we did not observe the effects of surgical or medication treatment in the two groups of patients, future work on this topic will provide more information and results regarding the post-treatment ratio between those two groups.

Ethics Committee Approval: Ethics committee approval was received for this study from the ethics committee of University of Montenegro Faculty of Medicine.

Informed Consent: Written informed consent was received from patients who participated in this study.

Peer-review: Externally peer - reviewed.

Author contributions: Concept - B.P., A.D., N.R.; Design - B.P., M.V.; Supervision - B.P., A.D., N.R.; Resource - B.P., A.D; Materials 
- B.P., M.V., N.R; Data Collection \&/or Processing - M.V., N.R.; Analysis \&/or Interpretation - M.V., N.R; Literature Search - M.V., B.P., N.R.; Writing - B.P., M.V.; Critical Reviews - B.P., A.D., N.R., M.V.

Conflict of Interest: No conflict of interest was declared by the authors.

Financial Disclosure: The authors declared that this study has received no financial support.

\section{REFERENCES}

1. Timpano M, Fontana D, Rolle L, Preto M, Falcone M, Sibona $\mathrm{M}$, et al. Sperm collection for medically assisted procreation in azoospermic patients. Urologia 2014;81(Suppl 23):27-31. [CrossRef]

2. Dada R, Kumar M, Jesudasan R, Fernández JL, Gosálvez J, Agarwal A. Epigenetics and its role in male infertility. $J$ Assist Reprod Genet 2012;29:213-23. [CrossRef]

3. Poongothai J, Gopenath TS, Manonayaki S. Genetics of human male infertility. Singapore Med J 2009;50:336-47.

4. Marconi M, Pilatz A, Wagenlehner F, Diemer T, Weidner W. Impact of infection on the secretory capacity of the male accessory glands. Int Braz J Urol 2009;35:299-308. [CrossRef]

5. Jungwirth A, Giwercman A, Tournaye H, Diemer T, Kopa Z, Dohle G, et al. European Association of Urology guidelines on Male Infertility: the 2012 Update. Eur Urol 2012;62:324-32. [CrossRef]

6. Haddad NG, Houk CP, Lee PA. Varicocele: a dilemma in adolescent males. Pediatr Endocrinol Rev 2014;2:274-83.

7. Pajovic B, Radojevic N, Vukovic M, Stjepcevic A. Semen analysis before and after antibiotic treatment of asymptomatic Chlamydia-and Ureaplasma-related pyospermia. Andrologia 2013;45:266-71. [CrossRef]

8. Yamamoto M, Hibi H, Hirata Y, Miyake K, Ishigaki T. Effect of varicocelectomy on sperm parameters and pregnancy rate in patients with subclinical varicocele: a randomized prospective controlled study. J Urol 1996;155:636-8. [CrossRef]

9. Unal D, Yeni E, Verit A, Karatas OF. Clomiphene citrate versus varicocelectomy in treatment of subclinical varicocele: a prospective randomized study. Int J Urol 2001;8:227-30. [CrossRef]

10. Marsman JW, Brand R, Schats R, Bernardus RE. Clinical and subclinical varicocele: a useful distinction? Eur J Obstet Gynecol Reprod Biol 1995;60:165-9. [CrossRef]

11. Valentino M, Bertolotto M, Derchi L, Pavlica P. Children and adults varicocele: diagnostic issues and therapeutical strategies. J Ultrasound 2014;17:185-93. [CrossRef]

12. Resim S, Cek M, Fazlioğlu A, Caşkurlu T, Gürbüz G, Sevin G. Echo-Colour Doppler Ultrasonography in the Diagnosis of Varicocele. Int Urol Nephrol 1999;31:371-82. [CrossRef]

13. Chiou RK, Anderson JC, Wobig RK, Rosinsky DE, Matamoros A Jr, Chen WS, et al. Color Doppler ultrasound criteria to diagnose varicoceles: correlation of a new scoring system with physical examination. Urology 1997;50:953-6. [CrossRef]

14. Feki NC, Abid N, Rebai A, Sellami A, Ayed BB, Guermazi M, et al. Semen quality decline among men in infertile relationships: experience over 12 years in the South of Tunisia. J Androl 2009;30:541-7. [CrossRef]

15. Bar-Chama N, Fisch H. Infection and pyospermia in male infertility. World J Urol 1993;11:76-81. [CrossRef]

16. Sanocka-Maciejewska D, Ciupińska M, Kurpisz M. Bacterial infection and semen quality. J Reprod Immunol 2005;67:51-6. [CrossRef]

17. Ma HG, Zhao WJ, Lu HK. Semen quality and sperm ultrastructure in infertile men with varicocele. Zhonghua Nan Ke Xue 2013;19:704-9.

18. Abdulmedzhidova AG, Kurilo LF, Shileǐko LV, Makarova NP, Klimova RR, Kushch AA. Asymptomatic genital herpes infection and infertility in males. Urologiia 2007;3:56-9.

19. Li H, Guo Y, Wang Y, Sun X. Genital Ureaplasma urealyticum infection in varicocele-related infertility. Chin Med $J$ (Engl) 1997;110:865-8. 